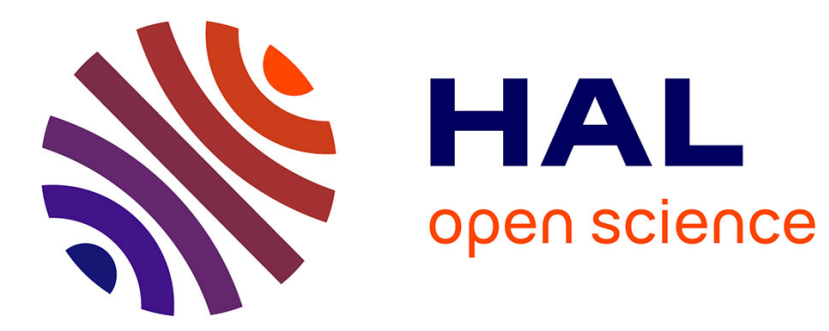

\title{
Projets en PPP, contrainte budgétaire et choix des investissements
}

\author{
Rémy Prud'Homme, Pierre Kopp
}

\section{To cite this version:}

Rémy Prud'Homme, Pierre Kopp. Projets en PPP, contrainte budgétaire et choix des investissements.

Revue d'Economie Politique, 2006, 116 (5), pp.601-611. halshs-00262433

\section{HAL Id: halshs-00262433 \\ https://shs.hal.science/halshs-00262433}

Submitted on 11 Mar 2008

HAL is a multi-disciplinary open access archive for the deposit and dissemination of scientific research documents, whether they are published or not. The documents may come from teaching and research institutions in France or abroad, or from public or private research centers.
L'archive ouverte pluridisciplinaire HAL, est destinée au dépôt et à la diffusion de documents scientifiques de niveau recherche, publiés ou non, émanant des établissements d'enseignement et de recherche français ou étrangers, des laboratoires publics ou privés. 


\title{
PROJETS EN PPP, CONTRAINTE BUDGÉTAIRE ET CHOIX DES INVESTISSEMENTS
}

\author{
Rémy Prud'homme \& Pierre Kopp ${ }^{1}$ \\ $1^{\text {er }} \operatorname{mars} 2006$ \\ JEL H43, H54
}

"Projets en PPP, contrainte budgétaire et choix des investissements" Revue $d^{\prime}$ Economie politique, $\mathrm{n}^{\circ} 3 / 2006$, à paraître.

\section{Résumé}

Le critère de la VAN constitue le pivot du choix d'investissement public. Certains projets font l'objet d'un partenariat entre le Public et le Privé (PPP). Le financement est alors mixte : subvention publique et investissement privé. Parmi un ensemble de projets, en présence d'une contrainte budgétaire, comment sélectionner le bon groupe de projets ? Le récent rapport Lebègue (2005) propose de retenir comme critère de choix non plus le bénéfice actualisé produit par l'investissement mais le bénéfice actualisé par euro public dépensé. Nous contestons cette approche qui ne prend en compte que la rentabilité du capital public et non à l'ensemble des ressources mobilisées. Nous testons la procédure de choix classique contre la procédure Lebègue dans le cas du choix entre les 17 projets autoroutiers proposés à un financement $P P P$ en 2005. La procédure Lebègue conduit à recommander à l'Etat d'investir dans plus de projets que la procédure classique, entraînant une grave mesallocation des ressources.

Mots clefs : PPP, TRI,

Projects selection under PPP

\footnotetext{
1 Professeur émérite, Université Paris XII, et Professeur, Université Panthéon-Sorbonne (Paris I).
} 
The VAN criteria constitute the criterium of choice for public investment. Some projects are subject to partnerships between the public and private sectors ( $P P P$ ). The financing is then mixed; public subsidy and private investment. In the presence of budgetary constraints, how to select the right group of projects from amongst all the projects? The recent Lebègue (2005) report suggests using as the selection criteria, not the greatest benefit produced by an investment but the benefit per public euro spent. We contest this approach which considers only the profitability of public capital and not the total of mobilised resources. We test the classic procedure against the Lebègue procedure in the case of the choice of the 17 autoroute projects proposed for PPP financing in 2005. The Lebègue procedure leads to recommending that the state invest in more projects than the classic procedure does, thus creating a serious misallocation of resources.

Keys words : PPP, IRR

\section{I - Introduction}

Pendant longtemps, les projets d'investissements publics, notamment en ce qui concerne les infrastructures, étaient doublement publics : financés par le contribuable et effectués par des entités publiques (ministères ou sociétés publiques). La question s'était donc posée de savoir quels projets, parmi les nombreux projets candidats, devaient être préférés. La réponse, donnée par la théorie du choix des investissements publics, était assez simple : les pouvoirs publics doivent préférer les projets qui ont la valeur actualisée nette (VAN) par euro investi la plus élevée ou le taux de rendement interne $\left(T_{R I}^{1}\right)$ le plus élevé. VAN et TRI à prendre en considération incluent tous les coûts et avantages des projets, y compris ceux qui sont externes et non monétarisés (qui doivent donc être monétarisés). Des débats existent sur la question de savoir si le critère de la VAN, qui implique un taux d'actualisation exogène, est préférable au TRI : c'est la position officielle française, récemment rappelée

\footnotetext{
1 Le TRI est une opération mathématique, qui résume en un seul nombre un flux de grandeurs positives et négatives actualisables (c'est le taux d'actualisation qui égalise à zéro la somme actualisée de toutes ces grandeurs). Ces grandeurs peuvent être financières, c'est-à-dire des dépenses et des recettes, ou encore économiques, c'est-à-dire des gains et des pertes économiques. Certains proposent d'appeler TRI le TRI appliqué à des grandeurs financières, et TRE le TRI appliqué à des grandeurs économiques. Il nous semble plus simple et moins ambigu de parler de TRI financier pour désigner le TRI appliqué à des grandeurs financières et de TRI économique (ou socio-économique) pour désigner le TRI appliqué à des grandeurs économiques.
} 
dans le rapport Lebègue (2005), mais pas celle qui prévaut dans certains autres pays et dans les organisations internationales. Lorsque l'Etat fait face à une contrainte budgétaire, ce qui est généralement le cas, il convient donc de classer les projets par ordre de VAN, ou plus exactement de VAN/euro investi (ou de TRI) décroissant, et de les prendre jusqu'à ce que la contrainte budgétaire soit saturée. Cette procédure fait l'objet d'un très large consensus (Cullis et Jones, 1998).

Depuis longtemps également, les investisseurs privés calculent le TRI financier (excluant les avantages ou les coûts externes) des projets qu'ils envisagent, et décident de faire ceux et seulement ceux qui ont un TRI plus élevé que le taux d'intérêt à long terme. Ils peuvent aussi calculer la VAN de ces projets avec un taux d'actualisation égal à ce taux d'intérêt à long terme. Des considérations relatives aux risques associés au projet, et aussi au ratio endettement sur fonds propres de l'entreprise peuvent compliquer le choix, mais là aussi la procédure fait l'objet d'un consensus.

Les choses se compliquent avec l'apparition et le développement des projets en partenariat public-privé (PPP), ou plus exactement des PPP avec subvention. Il est souvent intéressant de laisser au secteur privé le soin d'investir dans la création et l'exploitation d'infrastructures donnant lieu à redevances ou péages, comme par exemple une route, un pont, un réseau d'eau. Même lorsque les péages vont permettre de récupérer l'investissement -lorsque le TRI financier de l'ouvrage est jugé assez élevé par l'entreprise privée- on parle de PPP, parce que le droit de construire l'infrastructure a été donné par une entité publique, et parce que cette entité publique définit en général le niveau du péage. Il y a aussi des cas dans lesquels le TRI financier (au niveau de péage défini par l'entité publique) est insuffisant pour décider une entreprise privée à s'engager, mais pour lesquels il serait néanmoins socialement désirable qu'une entreprise privée s'engage. Dans ces cas, la solution consiste à ce que l'entité publique verse une subvention à l'entreprise privée, ce qui aura pour effet de remonter le TRI financier jusqu'au niveau considéré nécessaire par le privé. On pourrait dire de tels projets qu'ils sont doublement en PPP. L'entité publique intervient en effet doublement auprès de l'entreprise privée : en donnant l'autorisation et en fixant le niveau du péage d'une part, en subventionnant d'autre part. 
Le PPP avec subvention comme formule socialement désirable n'est pas une simple curiosité théorique. Un double gain lui est associé : le gain d'efficacité généralement lié à la gestion privée, le gain entraîné par une réduction $d u$ coût d'opportunité des fonds publics. Prud'homme (2004) et Piron (2004) ont pu montrer que cette formule pouvait ainsi être socialement préférable à des formules publiques pures ou publiques avec péage. En pratique, c'est la formule qui est actuellement envisagée pour bon nombre de projets d'investissements infrastructurels.

La question se pose alors de savoir quelle doit être le choix des pouvoirs publics devant un ensemble de projets en PPP avec subvention. Préférer des projets qui ont un taux de rentabilité sociale élevé mais qui demandent beaucoup de subventions, ou au contraire des projets qui ont un taux de rentabilité sociale plus faible mais qui demandent moins de subventions ? Avec une enveloppe budgétaire donnée, doit-on choisir peu de très bons projets à taux de subvention élevé ou beaucoup de bons projets à taux de subvention faible?

Le problème se pose très concrètement pour des institutions comme la Direction des Routes ou Réseau Ferré de France (RFF) qui suggèrent au gouvernement puis réalisent chaque année des projets qui se chiffrent en milliards d'euros. Le cas de la Direction des Routes correspond exactement au cas décrit ci-dessus. Celui de RFF est cousin. Les investissements de RFF sont financés à la fois par des subventions de l'Etat ou des collectivités locales (pour l'essentiel), et par les fonds propres de RFF. Est-ce que RFF doit préférer les investissements qui maximisent le rapport du bénéfice social à l'investissement total ou au contraire ceux qui maximisent le rapport de ce même bénéfice social à ses fonds propres ? Le problème ici n'est pas un problème de partenariat public-privé, mais de partenariat publicpublic. RFF joue ici le rôle du privé, mais RFF est une entité publique, motivée par le souci de l'intérêt général.

A cette question importante, une réponse officielle est apportée par un récent rapport du Plan, dit rapport Lebègue du nom du président du groupe d'experts qui l'a rédigé : " Pour tirer le meilleur parti [d'un] financement public limité, il convient de retenir comme règle de classement des projets non pas le bénéfice actualisé produit par l'investissement mais le bénéfice actualisé par euro public dépensé " (Lebègue 2005 p. 63, souligné 
dans le texte). Une contribution de Bonnafous et Jensen (2005a et $2005 b)$ préconise également cette solution.

Cette note propose un retour critique sur cette proposition afin d'en discuter la pertinence théorique et d'éclairer les conséquences de sa mise en œuvre. Elle commence par discuter les critères de choix des projets de PPP en situation de contrainte budgétaire (II), puis compare les conséquences des critères de choix concurrents sur l'échantillon de projets d'autoroutes déjà utilisé par Bonnafous et Jensen (2005a et 2005b) (III), avant de conclure (IV).

\section{II - Le choix entre projets de PPP subventionnés}

Le rapport Lebègue, on l'a vu, propose comme critère le choix de projets de PPP subventionnés en situation de contrainte budgétaire le "bénéfice actualisé par euro public dépensé ". Ce critère de choix est classique dans le cas des projets entièrement publics. Dans ces projets, financés par l'impôt, le total des euros publics dépensés est égal au total des euros dépensés, c'est-à-dire des dépenses, qui sont principalement des dépenses d'investissement. Pour simplifier l'expression, on considérera que ces dépenses sont uniquement des investissements. Le critère revient alors à classer les projets selon le rapport VAN/investissement. On décide d'engager les projets dans l'ordre du classement, jusqu'à ce que le total cumulé des investissements soit égal à la contrainte budgétaire. Dans la plupart des cas (non dans tous), le classement/choix ainsi obtenu est le même que le classement/choix selon les TRI socio-économiques. La question est de savoir si la procédure peut être extrapolée au cas des PPP avec subvention. Dans ce cas en effet, les "euros publics" dépensés en subvention ne sont qu'une partie de la dépense (d'investissement) totale, variable selon les projets. Le classement/choix selon le bénéfice actualisé par euro public dépensé est donc différent du classement/choix selon le bénéfice actualisé par euro dépensé.

L'exemple stylisé suivant éclairera le problème. L'entité publique est confrontée à 5 projets de PPP (A, B, $C, D, E)$ caractérisés par le montant de leur coût économique et social actualisé (le même pour chacun des projets), le montant des bénéfices économiques et sociaux, et la subvention nécessaire pour financer chacun de ces 
projets. On en déduit pour chacun des projets : le bénéfice net actualisé (c'est à dire la VAN), le bénéfice net actualisé par euro investi, et le bénéfice net actualisé par euro de subvention, c'est-à-dire par euro public dépensé.

Tableau 1 - Cinq projets de PPP

A

Dépenses actualisées

Bénéfice actualisé

Subvention requise*

Bénéfice net actualisé (VAN)

Bénéfice/euro investi

Bénéfice/euro public investi

* La corrélation entre le bénéfi est contre intuitif mais permet de mieux révéler le problème

La mise en œuvre des deux critères de choix va donner des résultats complètement différents, puisque l'ordre de classement des projets selon le critère du bénéfice par euro investi est dans l'exemple choisi exactement inverse du classement selon le critère du classement par euro public investi. Supposons une contrainte budgétaire égale à 40 . Le paquet de projets choisi doit être tel que le total des subventions soit égal ou inférieur à 40 . Le premier critère conduit à retenir les projets $D$ et $E$, ce que nous appellerons le choix a. Le second conduit à retenir les projets $A, B$ et $C$, ce que nous appellerons le choix b. Le tableau 3 indique les implications de ces choix.

Tableau 2 - Implications des critères de choix

\begin{tabular}{lrr}
\hline & $\mathrm{a}$ & $\mathrm{b}$ \\
Critère de choix & VAN/€ dépensé & VAN/€ public \\
Projets retenus & $\mathrm{D}, \mathrm{E}$ & $\mathrm{A}, \mathrm{B}, \mathrm{C}$ \\
Investissement total & 200 & 300 \\
Subvention (€s publics) & 40 & 40 \\
Investissement privé & 160 & 260 \\
VAN & 29 & 33 \\
VAN/euro dépensé & 0,145 & 0,110 \\
VAN/euro public dépensé & 0,725 & 0,825 \\
\hline
\end{tabular}

La règle de classement/choix du rapport Lebègue, représentée par b, correspond en effet à une VAN plus élevée (33 v. 29) et à une VAN moyenne par euro public dépensé plus élevée $(0,825 \mathrm{~V} \cdot 0,725)$.

Peut-on pour autant voir là un critère de décision publique satisfaisant ? Il nous semble que non, et que le critère du classement selon la VAN par euro total dépensé, 
c'est-à-dire a, doit être préféré. Mais dira-t-on, cela revient à faire deux projets au lieu de trois, à se contenter d'un bénéfice de 29 alors qu'on aurait pu obtenir un bénéfice de 33. Sans doute, mais il y a dans cette façon de voir une illusion d'optique, un tour de passe-passe. Cette présentation nous fait oublier que ce bénéfice de 29 économise 100 d'investissements privés supplémentaires.

Le paquet $\mathrm{b}$ produit effectivement une VAN plus élevée (de 14\%). Mais il le fait avec un investissement total également plus élevé (de $50 \%$ ). Les investissements publics (les subventions ou euros publics dépensés) sont bien les mêmes dans les deux cas, mais les investissements privés complémentaires sont bien différents (260 v. 160).

Préconiser la règle du classement selon la VAN/euro public dépensé revient à ignorer complètement le montant des investissements privés associés aux projets de PPP. Cette position n'est pas tenable. La décision publique a pour finalité le bien être social dans son ensemble, par l'allocation optimale des ressources rares. Les ressources rares à optimiser ne sont pas seulement les dépenses d'investissement public, mais aussi les dépenses d'investissement privé. La rareté de ces ressources doit être prise en considération. La façon la plus simple est de considérer le total de ressources rares consommées, c'est-à-dire le montant des investissements correspondant à chaque paquet de projets. C'est à ce montant total qu'il faut rapporter la VAN des projets de chaque paquet. La solution la plus avantageuse pour la société est celle qui est produite par le critère du classement des projets selon la VAN par euro dépensé -et non par euro public dépensé.

Le critère qui doit guider une entité publique, soucieuse de l'intérêt général de la nation, c'est bien celui du taux de rentabilité sociale, qui rapporte le bénéfice social net actualisé au montant total de l'investissement (et pas à la seule part de financement de l'un des partenaires). L'entité publique doit faire en sorte que soient réalisés les projets dont le TRI ou la VAN socio-économiques est le plus élevé, soit en les faisant elle-même, soit en les subventionnant, quel que soit le taux de subvention.

Le rapport Lebègue, bizarrement, ne donne aucune justification de son critère de choix et de classement -à notre avis erroné. Il se contente d'indiquer que "dans une situation de rareté des fonds budgétaires on démontre en effet que l'on maximise le surplus collectif en 
réalisant les projets suivant l'ordre de ce classement ". Mais il ne donne ni référence ni esquisse de " démonstration ". La proposition est vraie, et évidente, si les projets dont on parle sont intégralement financés par l'Etat ; dans ce cas "euros dépensés " et "euros publics dépensés " sont exactement la même chose puisque tous les euros dépensés sont publics (et l'adjectif "public " est alors explétif). Mais lorsque les projets dont on parle sont de PPP, ce qui est le cas qui nous occupe (et qui est le cas visé par le rapport Lebègue), la proposition cesse d'être vraie. L'extrapolation ou la généralisation n'est pas justifiée.

Le seul argument de sens contraire, a peine esquissé dans le rapport Lebègue, est l'abondance de liquidité dans le secteur privé. Il y aurait actuellement plus de capital privé à la recherche d'investissements rentables que d'investissements rentables disponibles, du moins en France. Le capital privé qui ne s'investit pas dans un projet de PPP ne s'investira nulle part, ou s'investira à l'étranger. Cela revient à dire que le coût d'opportunité du capital privé n'est pas égal au taux de rendement désiré et qu'il peut en quelque sorte être négligé. En d'autres termes, la seule rareté est celle des fonds publics, pas celle du capital, et il convient de solvabiliser l'investissement (comme on solvabilise la demande de logement par exemple). Cet argument, qui mérite discussion, ne nous apparâ̂t pas convaincant.

Tout d'abord, s'il était recevable, il serait contingent, et ne s'appliquerait qu'à la situation d'aujourd'hui et pas nécessairement à celle de demain.

De plus, un tel déséquilibre permanent du marché du capital est difficilement concevable. Il pèserait sur le taux de rendement désiré, et ramènerait celui-ci à un niveau plus modeste.

Enfin la théorie rappelle que le calcul économique repose toujours sur l'hypothèse de plein emploi des facteurs de production. Cette hypothèse est indispensable afin de pouvoir utiliser des données exprimées en prix et considérer qu'elles reflètent effectivement l'utilité sociale. Cette hypothèse peut parâ̂tre absurde dans un monde que caractérise précisément des déséquilibres constants des marchés et notamment du marché du travail. Mais le calcul économique relève d'un exercice en équilibre partiel où le reste de l'économie est supposé fonctionner de manière efficiente. L'objectif est alors d'identifier, dans un domaine donné, la solution la plus efficace, en ne prenant en compte que les distorsions du 
secteur considéré. Considérer que le capital privé est toujours en excès, comme le propose implicitement le rapport Lebègue, reviendrait à contredire cette hypothèse.

On peut voir un véritable Etatocentrisme dans la façon, prônée par le rapport Lebègue de rapporter la totalité du bénéfice d'un projet à la seule partie du coût supporté par l'Etat -et de mettre en avant les projets pour lesquels ce rapport est le plus élevé. On peut même y voir une certaine forme d'ignorance ou de mépris pour le secteur privé. La contribution de ce "partenaire " même lorsqu'elle est majoritaire, ne mérite pas d'être prise en compte. La société est au service de l'Etat, pas l'Etat au service de la société.

Notons au passage qu'une pratique comparable est courante dans le cas des financements publics multiples. Chaque entité publique (Europe, Etat, région, département, commune) qui co-finance un projet a tendance à comparer la totalité des bénéfices à sa seule contribution partielle. Elle considère le bénéfice actualisé net par son euro public dépensé. Il y a là une recette assez sure pour financer de mauvais investissements, c'est-à-dire des projets qui ne sont pas socialement rentables. Mais cette pratique dangereuse reste fondée sur une représentation politique vague, inexprimée. Elle n'est pas, comme dans le cas qui nous occupe, basée sur une théorie formalisée et revendiquée, bientôt traduite dans des circulaires ministérielles.

\section{IV - Le cas de 17 projets d'autoroutes}

$I=$ le montant de l'investissement, réalisé durant 1 'année 1 ,

$\mathrm{TE}=$ le TRI économique et social,

$\mathrm{TF}=$ le TRI financier,

$\mathrm{G}_{i}=$ les gains socio-économiques de $l^{\prime}$ année $i$, qui commencent pendant $l^{\prime}$ année 2 , avec $G_{i}=G_{2} *(1+2 \%)^{i}$,

$B_{i}=$ les bénéfices financiers de $l^{\prime}$ année $i$, avec $\mathrm{B}_{\mathrm{i}}=\mathrm{B}_{2} *(1+2 \%)^{\mathrm{i}}$,

$\mathrm{V}=\mathrm{la}$ VAN (valeur actualisée nette), au taux de de $4 \%$,

$\mathrm{S}=$ la subvention nécessaire pour assurer un $\mathrm{TF}$ égal à $10 \%$

$\mathrm{P}=$ le montant des investissements privés, avec $\mathrm{P}=\mathrm{I}-\mathrm{S}$, 
On calcule avec un tableur et par tâtonnement le flux $\mathrm{G}\left(\mathrm{G}_{2}, \mathrm{G}_{1}\right)$ au moyen de l'équation suivante, dans laquelle I et $\mathrm{TE}$ sont connus :

$$
I=\sum_{i} G_{2}(1,02)^{i} /(1+T E)^{i+1}
$$

Le flux $G$, ou simplement $G_{2}$, connu on en déduit la VAN à $4 \%$ :

$$
V=-I+\sum_{i} G_{2}(1,02)^{i} /(1,04)^{i+1}
$$

On calcule de la même façon le flux de bénéfices $B_{i}$ :

$$
I=\sum_{i} B_{2}(1,02)^{i} /(1+T F)^{i+1}
$$

Le flux $B_{i}$ calculé, on calcule l'investissement privé $P$ qui assure, avec ce flux, un TRI Financier (TF) égal à $10 \%$, au moyen de l'équation :

$$
P=\sum_{i} G_{2}(1,02)^{i} /(1,1)^{i+1}
$$

On en déduit la subvention $\mathrm{S}$ requise :

$$
S=I-P
$$

On produit ainsi le tableau 4 suivant 
Tableau 4 - Investissement I), TRI économique (TE) et financier (TF), VAN et subvention (S) requise de 17 projets autoroutiers français

\begin{tabular}{|c|c|c|c|c|c|}
\hline & $\begin{array}{r}I \\
(M €)\end{array}$ & $\begin{array}{c}\mathrm{TE} \\
\left(\frac{\circ}{0}\right)\end{array}$ & $\begin{array}{l}\mathrm{TF} \\
\left(\frac{\circ}{0}\right)\end{array}$ & $\begin{array}{r}\text { VAN } \\
(\mathrm{M} €)\end{array}$ & $\begin{array}{r}S \\
(M €)\end{array}$ \\
\hline Arles-Salon & 283 & $74 \%$ & $8 \%$ & 4606 & 104 \\
\hline A88 Caen-Argentan & 250 & $9 \%$ & $1 \%$ & 456 & 181 \\
\hline Amérieu-Bourgoin & 500 & $11 \%$ & $1 \%$ & 1095 & 365 \\
\hline Annemasse-Thonon & 378 & $18 \%$ & $8 \%$ & 1377 & 256 \\
\hline Dijon-Dole & 213 & $12 \%$ & $10 \%$ & 510 & 43 \\
\hline Dole-Bourg & 737 & $20 \%$ & $7 \%$ & 3053 & 267 \\
\hline Grenoble-Sisteron & 1880 & $17 \%$ & $3 \%$ & 6332 & 1250 \\
\hline Isle Adam-Amiens & 488 & $45 \%$ & $6 \%$ & 4745 & 224 \\
\hline Lyon-Balbigny & 770 & $11 \%$ & $1 \%$ & 1679 & 558 \\
\hline Orléans-Courtenay & 470 & $13 \%$ & $3 \%$ & 1219 & 300 \\
\hline Rouen-Alençon & 580 & $9 \%$ & $1 \%$ & 1055 & 416 \\
\hline Saintes-Rochefort & 290 & $20 \%$ & $3 \%$ & 1187 & 190 \\
\hline Sens-Courtenay & 197 & $15 \%$ & $10 \%$ & 591 & 37 \\
\hline Toulouse-Pamiers & 450 & $30 \%$ & $4 \%$ & 2850 & 262 \\
\hline Tours-Alençon & 698 & $28 \%$ & $4 \%$ & 4106 & 425 \\
\hline Tours-Vierzon & 800 & $19 \%$ & $4 \%$ & 3101 & 471 \\
\hline Troyes-Auxerre & 1350 & $4 \%$ & $9 \%$ & 1350 & 350 \\
\hline Total & 10334 & - & - & 39313 & 5699 \\
\hline $\begin{array}{l}\text { Sources et notes : } \\
\text { Jensen } 2005 a, \text { cita } \\
\text { calculs décrits au } \\
\text { de rentabilité int } \\
\text { ans. }\end{array}$ & $\begin{array}{l}\text { sur les trois } \\
\text { Brunel } 2003 \\
\text { exte, avec ur } \\
\text { le financier }\end{array}$ & $\begin{array}{l}\text { prem } \\
\text { Pour } \\
\text { taux } \\
\text { equis }\end{array}$ & $\begin{array}{l}\text { co } \\
\text { deus } \\
\text { tua } \\
10 \%\end{array}$ & $\begin{array}{l}\text { s: Bor } \\
\text { nières } \\
\text { ion de } \\
\text { une pe }\end{array}$ & $\begin{array}{l}\text { et } \\
\text { es : } \\
\text { taux } \\
\text { de } 30\end{array}$ \\
\hline
\end{tabular}

Les projets du tableau 4 sont classés par ordre alphabétique. Il apparâ̂t que tous les projets ont un TRI socio-économique supérieur à $4 \%$, le taux d'actualisation officiel français, et mériteraient d'être engagés. Malheureusement, la situation budgétaire est telle que l'Etat n'a pas les 10,3 milliards d'euros nécessaires à cet effet. L'Etat peut donc recourir au PPP. Mais il apparaît également qu'aucun des dix-sept projets n'a un taux de rentabilité interne financier assez élevé pour intéresser le secteur privé (deux ont un TRI proche de 10\%). Des subventions sont donc nécessaires. Le total des subventions que la réalisation de ces projets impliquerait s'élève à 5,7 milliards d'euros. Supposons que la situation budgétaire soit telle que l'Etat ne dispose pour les investissements routiers que de la moitié de cette somme, c'est-à-dire au plus de 2,8 milliards. Le problème est donc de choisir l'ensemble de projets le plus intéressant pour la France, en respectant cette contrainte de subventions de 2,8 milliards. C'est ici que les méthodes de classement des projets et de choix entrent en concurrence.

On peut d'abord classer les projets par ordre de TRI économique décroissant, et prendre les premiers jusqu'à ce que les subventions cumulées soient inférieures à 2,8 milliards. C'est la méthode "classique". On peut 
deuxièmement classer les premiers par ordre de VAN par euro de subvention décroissant, et prendre les premiers jusqu'à ce que les subventions cumulées soient inférieures à 2,8 milliards. C'est la méthode "Lebègue ". On peut enfin, troisièmement, classer les projets dans un ordre aléatoire, tel que par exemple l'ordre alphabétique du tableau 2 et faire jouer de la même façon la contrainte budgétaire (Alain Bonnafous note finement que cette procédure simule assez bien, hélas, un processus de choix purement politique $\left.{ }^{1}\right)$. Le tableau 5 présente les résultats de ces trois procédures.

Tableau 4 - Résultats de trois procédures de choix des projets sous contrainte budgétaire

Aléatoire Classique Lebègue

Classement selon ordre alphab. TRI éco décr. VAN/subv décr.

Nombre de projets

Investissement total

Subventions

Investissement privé

VAN

7
4241
2466
1775
17429

7

7429
4,11
7,06

$9 \%$

VAN/investissement total

VAN/subvention

TRI éco minimal

Source : Tableau 4 ci-dessus

$\begin{array}{rr}8 & 10 \\ 4124 & 4534 \\ 2199 & 2279 \\ 1925 & 2255 \\ 25099 & 26126 \\ & \\ 6,09 & 5,76 \\ 11,46 & 11,46 \\ 18 \% & 12 \%\end{array}$

4534

2279

2255

5,76

$12 \%$

La méthode aléatoire est, sans surprise, la plus mauvaise des trois. Ramenée à aux ressources totales investies ou aux seules ressources budgétaires investies, la VAN qu'elle produit est la plus faible. La comparaison entre les deux autres méthodes est plus intéressantes. Le hasard des chiffres fait qu'elles diffèrent assez peu. Cela provient de ce que les TRI économiques et les VAN par euro public investi se trouvent, en l'espèce, assez bien corrélés. La méthode Lebègue conduit à faire un peu plus de projets (10) (Arles-Salon, Isle Adam Amiens, Sens Courtenay, Dijon Dole, Dole Bourg, Toulouse Pamiers, Tours Alençon, Tours Vierzon, Saintes Rochefort, Anemasse Thonon) que la méthode classique (8), (Arles Salon, Isle Adam Amiens, Toulouse Pamiers, Tours Alençon, Dole Bourg, Saintes Rochefort, Tours Vierzon, Anemasse Thonon). L'investissement total est plus élevé (d'environ 10\%) et, par construction, un montant de subvention identique. En pratique, parce que le choix est discret, le montant de subvention n'est pas identique, mais légèrement supérieur (de $4 \%$ ) dans le cas de la procédure Lebègue.

\footnotetext{
${ }^{1}$ Cela ne veut pas dire que le choix politique échappe à toute rationalité. Il obéit à une rationalité politique. Mais du point de vue économique qui nous occupé ici le résultat de cette rationalité apparâ̂t comme aléatoire.
} 
Il s'ensuit que l'investissement privé est plus important (de 17\%) dans le cas de la procédure Lebègue. Au total, on voit que le paquet de projets de la procédure classique a un ratio VAN/euro de subvention plus élevé $(11,16$ contre 11,41$)$ que le ratio du paquet de la procédure classique. Mais que le paquet Lebègue a un ratio VAN/euro investi plus faible $(5,76$ contre 6,09) que le paquet classique. Ce résultat n'a évidemment rien de surprenant, et il n'est pas propre à l'exemple utilisé l $^{\prime}$

Respecter la contrainte budgétaire est certainement nécessaire. Mais cela ne signifie pas que l'Etat doit choisir les projets qui maximisent le ratio VAN par euro public dépensé dans le cas de projets en PPP. Il nous semble que la mission de l'Etat est de faire les choix qui (dans le respect des contraintes budgétaires) vont maximiser l'utilité sociale des investissements du pays, telle que la mesure le ratio VAN par euro total dépensé. Il y parvient avec la procédure classique.

\section{V - Conclusion}

Le développement des projets en PPP conduit à reposer la question du choix des investissements. Ce choix reste entièrement entre les mains de l'Etat, puisque c'est l'Etat qui décide d'un PPP, et que de plus dans la plupart des cas une subvention est nécessaire pour que le projet se réalise. L'Etat a donc besoin d'une règle pour choisir entre les nombreux projets concurrents, et d'une règle qui respecte les contraintes budgétaires puisque le taux d'actualisation du Plan ne remplit plus cette fonction. La règle classique, développée dans le cas des projets totalement financé sur fonds publics (avant l'apparition des PPP) était de classer les projets par ordre de TRI économique et social décroissant ou de ratio VAN sur investissement total décroissant, et de prendre les projets jusqu'à saturation de la contrainte budgétaire. Le rapport Lebègue a cru devoir rejeter cette règle, et lui substituer une autre règle : classer les projets selon le ratio VAN par euro public de subvention décroissant, et les prendre jusqu'à saturation de la contrainte budgétaire. On a essayé de montrer que cette nouvelle règle est du point de vue de l'économie nationale moins désirable que la règle classique -sans être pour autant préférable du point de vue des finances publiques puisque les deux règles respectent la même contrainte budgétaire.

Bonnafous et Jensen (2005, a) indiquent, a contrario, que le TRI est d'autant plus un meilleur critère que le TRI socio-économique, que la contrainte budgétaire est forte. 


\section{Références}

Bonnafous, Alain \& Jensen, Pablo. 2005a. "Ranking Transport Projects by their Socioeconomic value of Financial Internal Rate of Return ?". Transport Policy, 12 :pp. 131-136.

Bonnafous, Alain; Jensen, Pablo. 2005b. "Le cofinancement usager-contribuable et le partenariat public-privé changent les termes de l'évaluation des programmes d'investissement public ". Document aimablement communiqué par les auteurs.

Cullis, John \& Jones, Philip (1998) " Public Finance and Public Choice " Oxford University Press ;

Lebègue, Daniel, Hirtzman, Philippe \& Baumstark, Luc. 2005. Le prix du temps et la décision publique. Paris. La Documentation Française. 96p.

Piron, Vincent. 2004. " "La dimension économique du partenariat public-privé dans les transports". Transports. $\mathrm{N}^{\circ} 424$, pp.93-103.

Prud'homme, Rémy. 1997. "Le Financement des routes par des péages fiscaux". Revue Française de Finances Publiques. $\mathrm{N}^{\circ} 57$, pp. 160-180.

Prud'homme, Rémy. 2004. "Infrastructure and Developement ", paper prepared for the 2004 Annual Bank Conference on Developement Economics, Washington, The World Bank, May 3-4, 2004, publié dans : Bourguignon, François \& Boris Pleskovic, ed. 2005. Lessons of Experience. Oxford University Press and The World Bank, pp.153-180. 